\title{
Detection of DNA Sequence Based on Proton Reduction Catalyzed by Deposition of Platinum-Complexes
}

\author{
Tomoyuki Yasukawa, ${ }^{1,2}$ Yuya Yamashita, ${ }^{1}$ Daichi Nakayama, ${ }^{1}$ Seiichiro lijima, ${ }^{3}$ Fumio Mizutani ${ }^{1}$ \\ ${ }^{1}$ Graduate School of Material Science, University of Hyogo, 3-2-1, Kouto, Kamigori, Ako, Hyogo 678- \\ 1297, Japan, \\ mizutani@sci.u-hyogo.ac.jp \\ ${ }^{2} J S T-C R E S T, 5$, Sanbancho, Chiyoda, Tokyo 102-0075, Japan, \\ ${ }^{3}$ National Institute of Advanced Industrial Science and Technology, Tsukuba, 305-8566, Japan
}

\begin{abstract}
:
A novel electrochemical technique for the detection of DNA sequence has been developed by a simple electrochemical deposition of chloro-2,2':6',2"-terpyridine platinum (II) chloride dihydrate (Ptcomplex) on a glassy carbon electrode. The intercalation of a Pt-complex to a double-stranded DNA (ds-DNA), which is formed with the complementary recognition of probe DNA to target DNA, caused to suppress the electroreduction of the Pt-complex due to the steric hindrance and decrease of the diffusion coefficient. Thus, the catalytic current for reducing proton by the platinum deposited on the glassy carbon electrode decreased with the increase of the concentration of target DNA. An amplified response for measuring the current for reducing proton as compared with that for reducing the Ptcomplex itself could provide the highly sensitive measurement of ds-DNA.
\end{abstract}

Key words: platinum deposition, hybridization, intercalation, proton reduction, and current amplification

\section{Introduction}

The development of simple and sensitive determination of DNA with the specific sequence is of considerable practical interest for many years. Electrochemical behavior of the direct electron transfer of nucleobases was well studied by using the mercury [1], boron-doped diamond [2, 3] and diamond-like carbon electrode [4]. The spatial trapping to the capture DNA immobilized on the substrate and the modification with signal molecules (e.g. fluorescent and redox active species, and enzyme) have been usually applied to determine DNA concentration with the target sequence. A hybridization to form the doublestranded DNA (ds-DNA) involves the mediation [5] or intercalation [6] of electroactive species. However, in many cases, the capture DNA which recognize and trap DNA with the target sequence should be immobilized on the electrode surface.

Recently, we have reported on the electrolytic deposition of cisplatin and the application of the platinum deposition for the highly-sensitive determination of DNA [7]. This procedure based on the suppression of platinum deposition by the decrease of the free cisplatin with the complexation of cisplatin with DNA, while does not support the quantitative determination of DNA with specific sequences.

In this presentation, we propose a simple and sensitive technique for the determination of DNA sequence based on the combination of the intercalation of chloro-2,2,':6',2"-terpyridine chloride platinum (II) dehydrate (Pt-complex) into ds-DNA formed with hybridization and the catalytic reduction of proton with the platinum deposited on a glassy carbon (GC) electrodes. Figure 1 shows the the principle of the electrochemical determination of target DNA with specific sequence by the intercalation of Pt-complexes to the hybridized ds-DNA. In the absence of target DNAs, Pt-complex was reduced to deposit platinum on the glassy carbon electrodes, which follows the increase of the cathodic current corresponding to the electrochemical reduction of proton on the deposited platinum surface (Fig. 1 Left). The formation of ds-DNA by adding the DNA with the specific sequence causes the decrease of the concentration of the free Pt-complex by the intercalation and thereby decreases the catalytic current for proton due to the decrease of the rate for the platinum deposition. (Fig. 1 Right) 


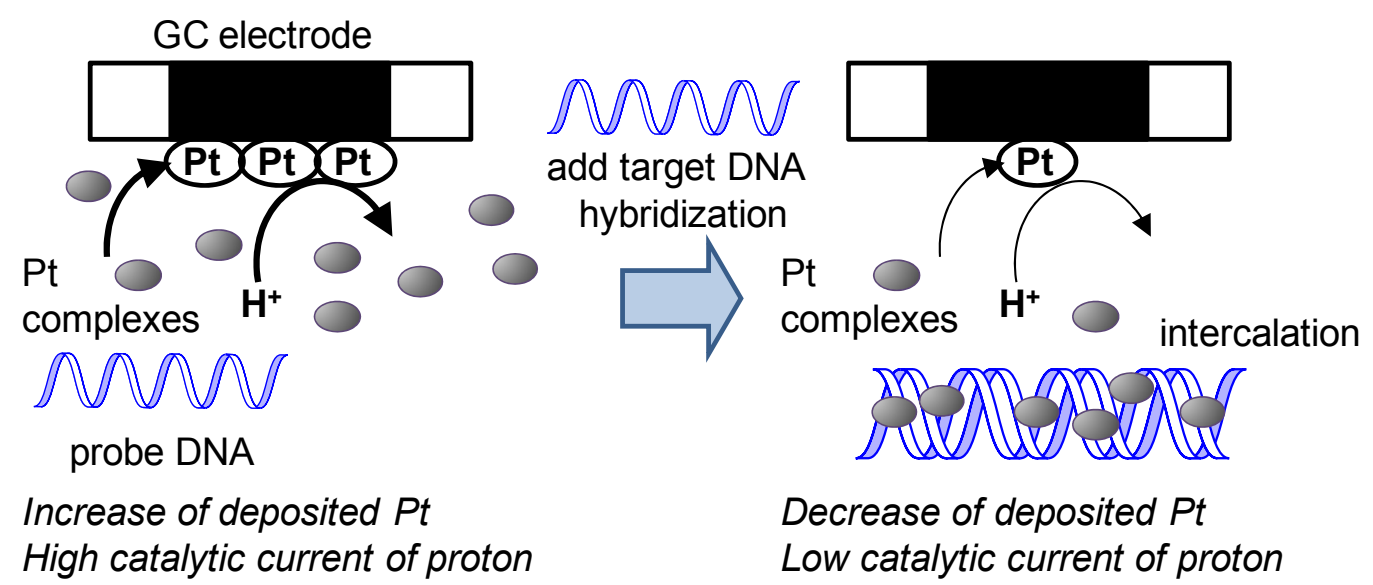

Fig. 1. Principle of the electrochemical determination of target DNA with specific sequence by the intercalation of Pt-complexes to the hybridized ds-DNA.

\section{Experimental Details}

Cyclic voltammetry was usually performed by sweeping the potential between 0.4 and $-1.5 \mathrm{~V}$ vs. $\mathrm{Ag} / \mathrm{AgCl}$ with the scan rate $50 \mathrm{mV} / \mathrm{s}$ in the buffer solution containing $0.1 \mathrm{mM}$ Pt-complex to determine the potential for the deposition of Ptcomplex.

The increasing rates of the catalytic current for proton by the deposited platinum were measured by amperometry with the stirring in the presence of the different concentration of target DNA. Pt-conplexes were synthesized according to published procedures [8]. Three synthetic oligonucleotides (20-mer) were used in this work and had the following sequences: 5'-TAT GGC TGG CTG GCT GGC AC-3' used as a target DNA, 5'-GTG CCA GCC AGC CAG CCA TA-3' used as a probe DNA and 5'-TAT GAC TGA CTG ACT GAC AC-3' used as a negative control DNA with non-complementary sequence, respectively. The different concentrations of target DNA were added to 0.1 $\mathrm{M}$ phosphate buffer ( $\mathrm{pH} 7.0$ ) containing $2.5 \mu \mathrm{M}$ probe DNA and 5.0 $\mu \mathrm{M}$ Pt-complex to allow to the hybridization and intercalation. After the incubation for $24 \mathrm{~h}$, the potential for the electrode was stepped to $-1.1 \mathrm{~V}$ from $0.4 \mathrm{~V}$ to deposit the platinum and measure the increasing rate of the reduction current for proton catalyzed by the deposited platinum.

\section{Results and Discussion}

We investigated the reduction potential for Ptcomplex on the GC electrode. Figure 1 shows the cyclic voltammograms for $0.1 \mathrm{mM} \mathrm{Pt}$ complex in $0.1 \mathrm{M}$ phosphate buffer $(\mathrm{pH} 7.0)$. The potential was swept at $50 \mathrm{mV} / \mathrm{s}$. For the first scan, the reduction current was slightly observed around $-1.0 \mathrm{~V}$ (Fig. 2A inset) and dramatically increased in negative potential region beyond $-1.0 \mathrm{~V}$ in the buffer solution containing Pt-complex (Fig. 2Aa), while no reduction response was obtained without complex (Fig. 2Ab). For the repeated scans, discernible increase of reduction current was given in negative potential region beyond -1.0 $\mathrm{V}$. After washing the electrode, the voltammetric measurement was performed in the same solution without Pt-complex. The similar voltammogram in the solution containing Ptcomplex was obtained in the free solution. These results strongly suggest that Pt-complex was reduced to deposit platinum on the GC electrodes by applying the potential $<-1.0 \mathrm{~V}$, which follows the appearance of large reduction current corresponding to the electrochemical reaction of proton on the deposited platinum surface. Thus, the measurement of the reduction current for proton is proved to be highly suitable for monitoring the deposition of Pt-complex, since the catalytic current for reducing proton is much larger than the current for the platinum deposition.

Figure 3 shows the variations of reduction currents in the presence of different concentration of Pt-complex. The potential was stepped from $0.4 \mathrm{~V}$ to $-1.1 \mathrm{~V}$ to reduce the complex. After step-like responses were observed, reduction currents were gradually increased. The increasing rate of reduction current decreased with decreasing the concentration of Pt-complex. The low concentration of Pt-complex during deposition should decrease the deposition rate of platinum, hence decreasing the increasing rate for the catalytic reduction of proton. Thus, the monitoring of the reduction current for proton is proved to be highly suitable for determining the concentration of Pt-complex, since the catalytic current for reducing proton is considerably much larger than the current for the platinum deposition (e.g., see Fig. 2A). 
(A)

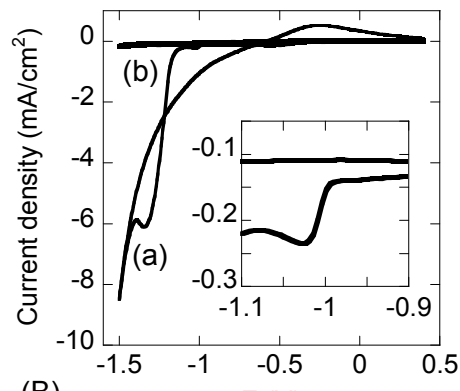

(B)

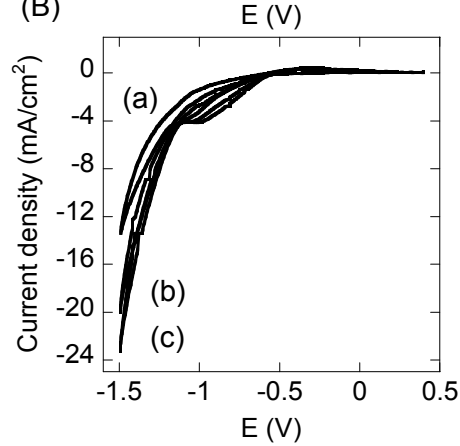

Fig. 2. Electrochemical properties for Ptcomplexes on the glassy carbon electrode by cyclic voltammetry. (A): Cyclic voltammograms of first cycle on the glassy carbon electrode (a) in the presence and (b) in the absence of 0.1 mM Pt-complexes. (B): Cyclic voltammograms of (a) second, (b) fourth and (c) sixth scan.

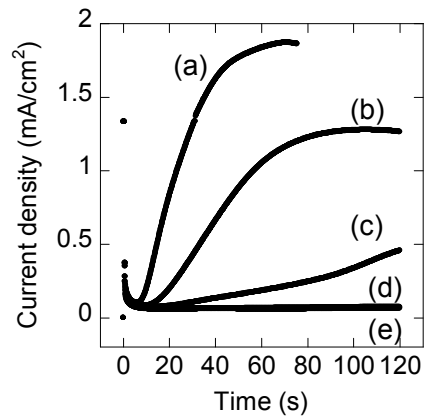

Fig. 3. Variations of reduction currents on GC electrode in $0.1 \mathrm{M}$ phosphate buffer containing Pt-complexes at $-1.1 \mathrm{~V}$. Concentrations of complexes are (a) 10, (b) 5.0, (c) 2.5, (d) 0.5 and (e) $0 \mu \mathrm{M}$.

The increasing rates of the catalytic currents were investigated by amperometry in the presence of the different concentrations of dsDNA from herring sperm. ds-DNA was added to the $0.1 \mathrm{M}$ buffer containing $5.0 \mu \mathrm{M}$ Pt-complex. After 90 min incubation, the potential of the GC electrode was stepped to $-1.1 \mathrm{~V}$ to deposit the platinum and measure the catalytic currents. Figure 4 shows the current-time curves obtained in the buffer containing the different concentrations of ds-DNA. The reduction currents are gradually increased because of the increase of the proton reduction with increasing the formation of platinum particles on the GC surface. In the solution without ds-DNA, the current reached the steady-state value $100 \mathrm{~s}$ after applying the potential. Furthermore, the increasing rate of the current significantly reduced with increasing ds-DNA concentration up to $50 \mu \mathrm{M}$. A concentration at least as low as $50 \mathrm{nM}$ can be detected in the present system. Pt-complexes were intercalated into added dsDNA, leading to decreased the concentration of free-complex. The decrease of deposition rate of platinum would be caused to the steric hindrance and increase of the diffusion coefficient of Pt-complex by the intercalation to ds-DNA.

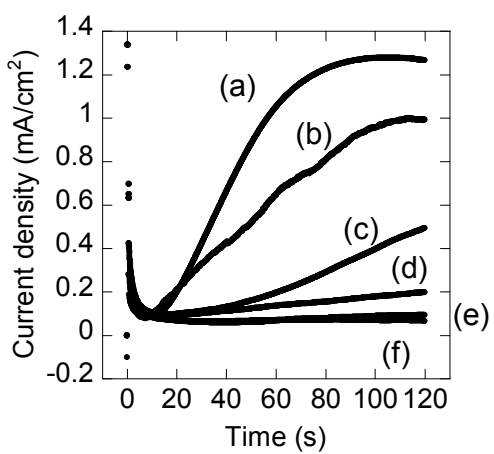

Fig. 4. Variations of reduction currents on GC electrode (a-e) in the presence of $5.0 \mu \mathrm{M} \mathrm{Pt}$ complexes containing ds-DNA (concentration: (a) 0, (b) 0.05, (c) 0.5, (d) 5.0 and (e) $50 \mu \mathrm{M}$ ) and $(f)$ in the absence of Pt-complex.

The increase of the reduction current was investigated to determine the concentration of target DNA. Figure 5A shows the amperometric current responses in $0.1 \mathrm{M}$ buffer solution containing $2.5 \mu \mathrm{M}$ probe DNA and different concentrations of target DNA. In the absence of target DNA, the reduction current drastically increased after the potential was stepped to $-1.1 \mathrm{~V}$ because of the increase of the proton reduction with increasing the platinum surface (Fig. 5b). The current-time curve obtained with only probe DNA is similar to that obtained with only Pt-complex (Fig. 5a). The result provably indicated that the Pt-complex does not interact with the single-stranded DNA (ss-DNA).

The addition of target DNA actually brought about a delay for the increase in the reduction current (Fig. 5c - 5g). Pt-complexes were intercalated into the ds-DNA formed by the complementary recognition event between the probe DNA and the target DNA added in the solution, and thereby decrease of the concentration of free Pt-complex. However, increasing rate obtained in the solution added DNA with non-complementary sequence is comparable with that in the solution without 
target DNA (Fig. 5Ah and 5Ab), because of the unprovided ds-DNA for intercalation. Thus, the presence of ss-DNA with non-complementary sequence does not inhibit the platinum deposition by decreasing the concentration of free complex. These results suggest that the DNA concentration can be determined from the current-time curve for the reduction of proton.
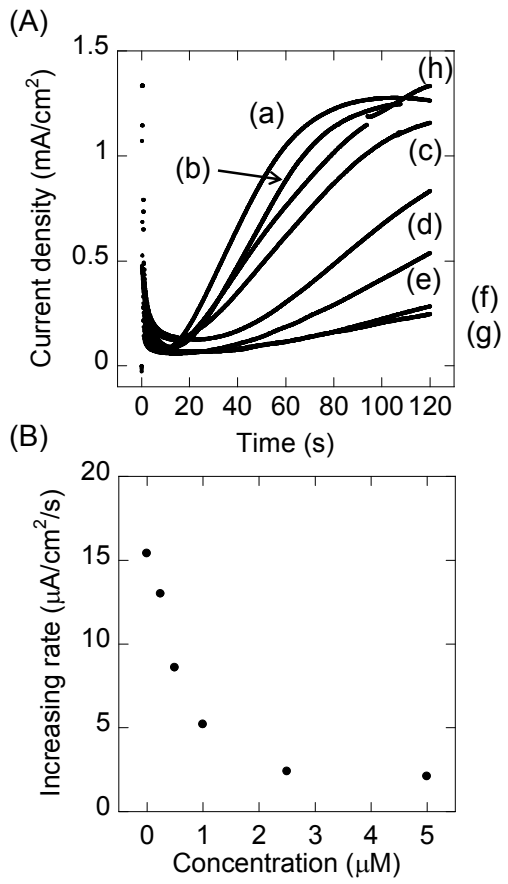

Fig. 5. (A) Current-time curves in $0.1 \mathrm{M}$ phosphate buffer containing $5.0 \mu \mathrm{M}$ Pt-complex, $2.5 \mu \mathrm{M}$ probe DNA and the different concentrations of target DNA. (a) Response obtained with only $5.0 \mu \mathrm{M}$ Pt-complex. Concentrations of target DNA are (b) 0 , (c) 0.25 , (d) 0.5, (e) 1.0 (f) 2.5 and (g) $5.0 \mu \mathrm{M}$. (h) Response obtained in the presence of $2.5 \mu \mathrm{M}$ ss-DNA with non-complementary sequence to the probe DNA. (B) Relationship between the target DNA concentration and the average increasing rates of reduction currents obtained 60-70 s after applying the potential.

Figure 5B shows the relationship between the target DNA concentration and the average increasing rates of reduction currents obtained 60-70 s after applying the potential. The reduction current decreased with the increase of the DNA concentration up to $2.5 \mu \mathrm{M}$ and saturated in the higher concentration due to the limitation of the formation of ds-DNA. The significant difference of the increasing rate was observed in $0.25 \mu \mathrm{M}$ target DNA. The measurement of the catalytic reduction current for proton actually resulted in the simple and sensitive determination of target DNA with complementary sequence.

\section{Conclusion}

We have demonstrated that DNA with the target sequence can be electrochemically detected on the basis of the intercalation of Pt-complexes into the ds-DNA which formed by the hybridization between the target and probe DNAs. The electrocatalytic current for proton reduction which is catalyzed by the platinum deposition decreased with the decrease of free $\mathrm{Pt}$-complex and suppression of the reduction of Pt-complex intercalated due to the steric hindrance. The present procedure yields a simple determination of DNA with specific sequence without the necessity of immobilization of capture DNA.

\section{Acknowledgements}

This work has been partly supported by Adaptable and Seamless Technology transfer Program (AS231Z01304C) from the Japan Science and Technology Agency.

\section{References}

[1] E. Palecek, Oscillographic Polarography of Highly Polymerized Deoxyribonucleic Acid, Nature 188, 656-657 (1960); doi: 10.1038/188656a0

[2] E. Palecek, M. Fojta, Detecting DNA hybridization and damage, Anal. Chem. 73, 74A-83A (2021); doi: 10.1021/ac0123936

[3] C. Prado, G. -U. Flechsig, P. Gründler, J. S. Foord, F. Marken R. G. Compton, Electrochemical analysis of nucleic acids at boron-doped diamond electrodes, Analyst 127, 329-332 (2002); DOI: 10.1039/B111548K

[4] O. Niwa, J. Jia, Y. Sato, D. Kato, R. Kurita, K. Maruyama K. Suzuki, S. Hirono, Electrochemical performance of angstrom level flat sputtered carbon film consisting of $\mathrm{sp} 2$ and $\mathrm{sp} 3$ mixed bonds, J. Am. Chem. Soc. 128, 7144-7145 (2006); DOI: 10.1021/ja060609I

[5] D. H. Johnston, K. C. Glasgow, H. H. Thorp, Electrochemical Measurement of the Solvent Accessibility of Nucleobases Using Electron Transfer between DNA and Metal Complexes, $J$. Am. Chem. Soc. 117, 8933-8938 (1995); DOI: 10.1021/ja00140a006

[6] K. Hashimoto, K. Ito, Y. Ishimori, SequenceSpecific Gene Detection with a Gold Electrode Modified with DNA Probes and an Electrochemically Active Dye, Anal. Chem. 66, 3830-3833 (1994); DOI: 10.1021/ac00093a045

[7] Y. Yoshimoto, T. Yasukawa, Fumio Mizutani Cisplatin-based DNA sensing with enhanced current response, Analyst,134, 2113-2117 (2009); DOI: 10.1039/b906734e

[8] G. T. Morgan, F. H. Burstall, Researches on residual affinity and co-ordination. Part XXXV. 2 : 2' : 2"-Tripyridylplatinum salts J. Chem. Soc. 1498-1500 (1934); DOI: 10.1039/JR9340001498 